\title{
Impact du modèle Shareholder sur la stratégie de croissance de la firme globalisée
}

\author{
Mme Fatma Braham ${ }^{1}$
}

Résumé : Compte tenu d'un environnement économique globalisé - privatisations, financement sur fonds propres et exacerbation de la concurrence internationale - les modalités de croissance, de contrôle et de gestion des entreprises, ainsi que les stratégies d'internationalisation qu'elles vont mettre en ouvre se sont, sensiblement transformées.

Le propos du présent papier n'est pas seulement d'envisager l'évolution de l'étroite relation entre la sphère productive et la sphère financière ou de décrire l'ampleur prise par la financiarisation de l'économie au cours des dernières années, il témoigne au contraire d'une profonde transformation au sein de l'organisation productive de l'entreprise globalisée, on souligne une reconfiguration de ces organisation qui a été dictée par un nouveau modèle de gouvernance de ces entreprises.

Ainsi, ce papier montre un impact loin d'être négligeable de l'adoption d'un nouveau modèle de gouvernance de l'entreprise globalisée sur les stratégies de croissance interne et externe. Cet impact renforce la contradiction entre, d'une part, la société d'actionnaires représentant les intérêts de la finance et d'autre part, l'entreprise et ses finalités productives.

Mots clés : globalisation financière, économie financiarisée, gouvernance d'entreprise.

\section{Introduction}

Déréglementation et libéralisation des marchés financiers, privatisations des entreprises, prédominance du rôle des fonds propres dans le financement des entreprises, exacerbation de la concurrence à l'échelle mondiale, etc. ; autant de composantes centrales de la globalisation financière ${ }^{2}$. Tous ces facteurs ne vont pas sans produire des bouleversements majeurs au sein des organisations productives des firmes globalisées. Ces transformations reconnues tout au long de ces trente dernières années, d'une part, au niveau des modalités de direction et de contrôle des entreprises et d'autre part, à travers le choix de stratégies productives bien spécifiques.

Ainsi, la globalisation recouvre des politiques économiques spécifiques, des réformes financières, des institutions économiques internationales, un accroissement de l'internationalisation des échanges de biens et de services, des modifications de la structure du capital des entreprises, des stratégies et organisations productives typiques ou encore une progression de l'incertitude de l'activité.

Souvent, cette globalisation est assimilée à une force quasi naturelle et extrapolitique. De telles sortes que l'on éprouve les plus grandes difficultés à distinguer ce à quoi elle constitue précisément dans la réalité et à évaluer ses conséquences sur la sphère productive.

Pourtant, la globalisation paraît générer un certain nombre de manifestations directes sur les entreprises qui sont loin d'être réductibles à sa dimension purement financière. Ces transformations caractérisées essentiellement par la dominance d'un nouveau modèle de gouvernance « shareholder» ont donné jour à une multiplication de la stratégie de «fusion-acquisition » traduite par des opérations de Re-engineering et de soustraitance mais aussi à des restructurations des activités industrielles et des réductions d'effectifs, donnant jour à un nouveau modèle de croissance des entreprises globalisées.

Ainsi, l'objectif de ce travail est de décrire les expressions concrètes d'un nouveau modèle de gouvernance à travers les grandes entreprises. Il s'agira dans un premier temps de caractériser l'environnement économique dans lequel opèrent les entités industrielles globalisées, dans un second de montrer comment s'est transformée la structure de financement des entreprises suite aux changements du contexte économique dans lequel elles évoluent (cadre précisé dans la section précédente). Ensuite, une section sera consacrée à la spécification du modèle Shareholder (section 3) et une quatrième section tentera de contourner les retombés de ce modèle sur les stratégies de croissance externe des firmes globalisées.

\footnotetext{
${ }^{1}$ Maître- assistante à l'Ecole Supérieure de Commerce de Tunis. Département Economie et Méthodes Quantitatives. Université deLa Manouba. Tunisie.

${ }^{2}$ La globalisation financière est le nom donné à des transformations qui ont affecté les principes de fonctionnement de la finance. Ce sont des transformations très profondes qui associent étroitement la libéralisation des systèmes financiers nationaux et l'intégration internationale (Aglietta, 2000).
} 


\section{Processus de la globalisation financière : vers une économie financiarisée}

A partir du milieu des années 1960, un passage d'une «configuration internationale » des grandes firmes à une «configuration globale», a été repéré par nombres d'auteurs notamment C.A. Michalet (2002). C'est à partir de cette période que date le franchissement des premières étapes de libéralisation et de déréglementation - adoptées par les principaux pays industrialisés -, conduisant à l'avènement d'une vision globale de la part des entreprises fortement internationalisées.

Toutefois, il faut signaler une phase de transition qui a annoncé l'émergence de la phase de globalisation. C.-A. Michalet (2002) parle d'une « configuration multinationale » de la mondialisation notant la prédominance des investissements directs à l'étranger (IDE) à travers une forte mobilité des activités économiques en dehors des pays d'origine (ce mouvement a touché d'abord les multinationales américaines et britanniques, puis ensuite les multinationales européennes à partir du milieu des années 1970 et suivies récemment par les multinationales originaires des pays émergents, à savoir celles de l'Amérique latine et du sud est asiatique).

Cette configuration a été le résultat d'une exacerbation d'une concurrence oligopolistique, la compétitivité par les prix et par les coûts devient alors un élément clé de la logique économique en place. Cela conduit les entreprises à mettre en œuvre différentes stratégies de délocalisation, à l'image de la stratégie de marché qui consiste à implanter des filiales relais orientées exclusivement ou majoritairement vers la vente de produits destinés à des marchés locaux (nationaux ou régionaux). Premier signe de rupture avec la «configuration internationale», annonciateur de la globalisation: les firmes multinationales occupent désormais une place centrale dans l'économie.

Ainsi, on passe progressivement d'un système international où les pouvoirs publics et les gouvernements représentants les intérêts économiques de leurs entreprises dominent les relations commerciales internationales à un système où les entreprises multinationales vont davantage intervenir sur les décisions publiques. Puis surtout, la transformation du système monétaire international qui va accélérer à la marche vers la mondialisation financière.

En août 1971 avec les accords de Bretton Woods, la mutation du système monétaire international via le démantèlement du « Gold Exchange Standard » et la création du principe dit des « taux de changes flexibles » marque une étape décisive (Chesnais, 1996) dans le processus qui a mené à la configuration globale de la mondialisation.

Désormais, cette réglementation a été au profit des marchés financiers qui vont devenir les nouveaux régulateurs des systèmes de paiements internationaux. Le cours des devises nationales est déterminé par le jeu de l'offre et la demande, à l'instar de n'importe quelle marchandise. Ainsi, en cherchant à tirer profit des variations des différents taux des devises entre les places financières internationales, les institutions financières privées deviennent les instances de régulation principales du marché des changes.

Cependant, elles devront encore attendre la déréglementation des mouvements de capitaux au cours des années 1980 avant d'être en mesure de développer pleinement leur activité spéculative. Cette dérégulation intervient à travers le réajustement et l'affirmation politique de l'orientation économique internationale qui constitue une autre étape fondamentale vers la globalisation.

Dans un contexte de ralentissement de la croissance mondiale et de remise en question des politiques keynésiennes qui avaient prévalu depuis 1945, les équipes gouvernementales américaines et britanniques inaugurent une nouvelle politique économique d'inspiration monétariste au tournant des années 1980.

D'une part, cette nouvelle doctrine économique favorise l'accentuation de la déréglementation financière. Pour y parvenir, elle conduit à alléger entre autres et significativement les législations, les réglementations et le contrôle des transactions. Le décloisonnement et la déréglementation du marché des actions viennent compléter celui des devises tandis que les marchés financiers, les organisations financières non bancaires (fonds de pension, sociétés d'investissement collectif, OPCVM, etc.) deviennent des sources de financement dominantes aux détriments de l'emprunt bancaire.

D'autre part, le credo monétariste pose le marché comme principe exclusif de régulation par opposition à l'ingérence de l'État dans l'économie. Elle pousse à la réduction de l'interventionnisme étatique et favorise l'initiative privée en encourageant la privatisation de l'économie et des capitaux des entreprises.

En réduisant le pouvoir des États, les détenteurs de capitaux visent alors à accroître le leur. De cette façon, un système où la décision publique et politique prévaut est remplacé par un pouvoir censitaire où se sont ceux qui possèdent la puissance financière qui maîtrisent par voie de conséquence le pouvoir politique. Ces éléments caractérisent une économie financiarisée qui a un impact important à la fois sur la structure capitalistique des grands groupes mais aussi sur la structure productive de ces groupes. 


\section{Les principales transformations des structures de financement des entreprises dans une économie financiarisée}

Les mutations du mode de financement des entreprises dans le contexte de la globalisation financière peuvent être présentées comme le passage d'un régime d'endettement bancaire, majoritaire, à un régime de fonds propres. Cela signifie en substance que les entreprises se financent désormais de plus en plus sur leurs fonds propres, au détriment de l'endettement bancaire. On parle également de désintermédiation bancaire pour qualifier cette tendance qui touche les modes de financement des entreprises.

Plus précisément, les entreprises ont utilisé, de façon croissante depuis la fin des années 1970, deux moyens privilégiés pour constituer leurs ressources financières propres.

Le premier est le recours massif aux marchés financiers sur lesquels les entreprises émettent des titres de propriété de leur capital - actions ou obligations - qui leur permettent de trouver des investisseurs censés accroître leur capital. L'offre de titres de propriété a ainsi considérablement progressé, que ce soit par l'augmentation du nombre de sociétés cotées en Bourse ou bien par l'élévation des émissions d'actions. Cette dernière a été significative pour le cas de la France par exemple puisque le montants des actions en circulation est passé de 7,9 milliards d'euros en 1980 à 113,7 milliards en 2000 (Aglietta et Rébérioux, 2004), malgré un recul à 78 milliards en 2004 (Baumel et al., 2005). On saisit alors le rôle décisif qu'ont pu jouer les mesures de libéralisation des marchés financiers et de privatisation des entreprises depuis le début des années 1980 en incitant les entreprises à mobiliser des capitaux sur les marchés financiers.

Le second moyen réside dans les capacités d'autofinancement des entreprises qui, elles aussi, ont connu une hausse vertigineuse durant la décennie 1990. Alors qu'elles représentaient 28 milliards d'euros en 1980 (Plihon, 2004), les capacités d'autofinancement des entreprises françaises s'élèvent à 226 milliards en 2003 (Insee, 2005). La hausse record du recours à l'autofinancement est liée à la fois à la progression des résultats des entreprises, qui permet d'accumuler une épargne abondante, et à un ralentissement du volume des investissements. La part de ces derniers dans la valeur ajoutée est ainsi passée de 22\% en 1970 à 18,6\% en 2004 (Loué, 2005).

En fait, de façon globale, c'est surtout l'augmentation sensible des profits au cours des trente dernières années qui s'est révélée être la condition, au niveau des entreprises, de l'accumulation des fonds propres et de leur utilisation en tant que source principale de financement. De fait, en Europe, le taux de profit ${ }^{3}$ atteint 21,5\% en 1996 alors qu'il avait chuté à environ 12\% en 1980 (Duménil, Lévy, 2000). La hausse des profits des entreprises à tout d'abord permis de rendre attractif leurs titres de propriété sur les marchés financiers en offrant aux actionnaires des rémunérations conséquentes. Puis elle a également œuvré en faveur de l'autofinancement en dégageant de l'épargne d'entreprise.

A ce titre, M. Aglietta et A. Rébérioux (2004) repèrent deux fonctions remplies par les marchés financiers qui se sont particulièrement développées ces dernières années. La première consiste à faciliter et à multiplier les opérations de croissance externe des entreprises en finançant les fusions acquisitions grâce à l'échange de titres sur les marchés. Pour mener à bien de telles stratégies industrielles dont l'objectif est d'acquérir les capitaux et/ou les compétences d'entreprises concurrentes ou partenaires, il a fallu alors être favorablement reconnu sur les marchés financiers.

La deuxième fonction de l'émission d'actions en Bourse par les entreprises est la mise en place de nouveaux systèmes de rémunération fondés sur la possibilité, pour les salariés, d'acquérir des titres de propriété de leur entreprise grâce à l'actionnariat salarié et la distribution d'actions à tarif préférentiel pour les cadres dirigeants (stock-options). Il s'agit là d'une stratégie visant à fidéliser le personnel et à conserver des compétences qui peuvent s'avérer rares dans certains secteurs. Donc là encore, la fonction des marchés financiers revêt une dimension éminemment stratégique aux yeux des dirigeants (Aglietta et Rébérioux, 2004).

Par ailleurs et dans une moindre mesure, T. Coutrot (1999) discerne un troisième rôle des marchés financiers que l'on pourrait qualifier d'officieux, qui consiste à être utilisés par les entreprises comme instrument disciplinaire envers les salariés. Ainsi, le prétexte d'une pression inexorable exercée par des actionnaires «lointains » est effectivement avancé pour justifier une intensification du travail des salariés ou bien leur imposer des restructurations récurrentes pouvant se traduire par des licenciements. La cotation d'une entreprise en Bourse permet alors de normaliser - voire de naturaliser - les niveaux de rentabilité exigés.

\section{Le modèle du «shareholder » et la « gouvernance d'entreprise »}

Les rapports entre les trois principaux acteurs de l'entreprise - actionnaires, salariés et dirigeants - ont été fortement modifiés suite à la financiarisation de l'économie. Ainsi, et avec les vagues de privatisation du capital des entreprises et le développement du financement par fonds propres depuis le début des années 1980,

\footnotetext{
${ }^{3}$ Gérard Duménil et Dominique Lévy définissent de la manière suivante le taux de profit : « Le taux de profit est l'indicateur de la rentabilité du capital. Il rapporte la masse des profits réalisés au cours d'une période donnée, une année, à la somme totale des fonds investis dans une entreprise, un secteur ou l'ensemble de l'économie » (Duménil, Lévy, 2000, p. 34).
} 
le pouvoir des actionnaires a été largement renforcé au détriment de celui des dirigeants et, a fortiori, de celui des salariés.

On assiste alors à la fin du «capitalisme managérial» qui certes, reposait sur la division entre détenteurs du capital et possesseurs de la force de travail, mais qui laissait cependant envisageable la négociation d'accords entre dirigeants et salariés en matière de partage des gains de productivité, garantissant ainsi une certaine stabilité dans la répartition de la valeur ajoutée.

Avec l'émergence du «capitalisme actionnarial» ou «patrimonial», on passe du modèle de «stakeholder» qui concevait l'entreprise comme une communauté d'intérêts entre ses trois partenaires (actionnaires, dirigeants et salariés) au modèle de «shareholder » qui privilégie la satisfaction des intérêts des actionnaires. Le pouvoir des actionnaires s'est vu, en outre, renforcé par le développement de la gestion collective de l'épargne avec la montée en puissance des investisseurs institutionnels.

Alors qu'auparavant, les actifs financiers étaient répartis entre de nombreux épargnants individuels, ils sont désormais gérés par des fonds d'investissement et dès lors concentrés au cœur d'une minorité d'investisseurs institutionnels (Batsch, 2002).

Selon les classifications habituelles, les investisseurs institutionnels regroupent trois types principaux d'institutions : les organismes de placement collectif (OPC), les compagnies d'assurances et les fonds de pension ${ }^{4}$.

Au centre des relations de gestion collective de portefeuille se trouve un lot d'actifs dont le rendement est partagé entre plusieurs catégories d'acteurs: les propriétaires des actifs, les commanditaires, et les professionnels qui gèrent les portefeuilles pour le compte des premiers. Les règles qui déterminent la répartition des risques et des rendements entre ces acteurs diffèrent selon les institutions.

La redistribution du capital des entreprises par le truchement de l'épargne salariale et des investisseurs institutionnels ne semble pas présager une nouvelle démocratie actionnariale comme le laissent entendre certains de ses promoteurs (Manière, 1999). Tout au contraire, ce nouveau mode de gestion collectif de l'épargne actionnariale augure une appropriation et une concentration privée du capital inédites par l'intermédiaire notamment des investisseurs institutionnels (Lordon, 2000).

A cette nouvelle conception de l'entreprise incarnée par le modèle de «shareholder » qui consacre les pleins pouvoirs aux actionnaires et aux investisseurs institutionnels va correspondre un nouveau principe orchestrant le contrôle et la conduite des firmes ainsi que les pratiques managériales. Il s'agit de la «corporate governance » que l'on préfèrera traduire par «gouvernance d'entreprise » plutôt que "gouvernement d'entreprise ${ }^{5} »$.J. Nikonoff (2000) fournit une définition concise de ce qu'il faut entendre par «gouvernance d'entreprise » : elle désigne «l'action des fonds de pension américains, particulièrement des fonds de pension publics, qui vise à maximiser les rendements des actions des entreprises dont ils sont actionnaires.

Pour atteindre cet objectif, les fonds de pension tentent de contrôler les entreprises et leurs équipes dirigeantes afin qu'ils adaptent la gestion à leur demande » (Nikonoff, 2000, p. 118). L'évaluation de la gouvernance d'une entreprise va alors s'effectuer à l'aune de cinq critères qui portent respectivement sur la qualité de l'information recueillie par les actionnaires, les droits des actionnaires faisant correspondre à une action une voix, la constitution du conseil d'administration, l'absence d'obstacles aux décisions d'offres d'achats hostiles - susceptibles d'exercer une pression sur les dirigeants - puis enfin, les modalités de rémunération des dirigeants qui prennent très souvent la forme de stock-options (Plihon, 2000 ; Finet, 2005).

La «gouvernance d'entreprise » est donc orientée vers la maximisation de la valeur boursière d'une entreprise ou encore, ce qu'il convient d'appeler la « shareholder value» (la valeur actionnariale) (Lazonick, O’Sullivan, 2000 ; Caby, Hirigoyen, 2005). Ces dernières années, la « création de valeur pour l'actionnaire » a connu un certain retentissement dans les grandes entreprises en étant notamment formalisée par le ratio EVA (Economic Value Added $)^{6}$. Ce ratio constitue par exemple la référence centrale légitimant l'existence de la convention boursière imposant le taux de rentabilité du capital - le ROE (Return On Equity) - à 15\% (Plihon, 2002).Par conséquent, dans un contexte de concurrence internationale exacerbée et avec ce nouveau mode de contrôle des entreprises par les actionnaires et les investisseurs institutionnels, l'entreprise globalisée est considérée comme un actif dont il faut accroitre la valeur boursière plutôt qu'une entité orientée vers la production de biens et de services. Une telle conception de l'entreprise sous le règne du «capitalisme

\footnotetext{
${ }^{4}$ Le poids de ces différentes institutions financières est très variable selon les pays. Les fonds de pension dominent aux États-Unis tandis que les assurances ont le plus gros portefeuille au Japon ; en France, la première place revient aux OPCVM (Organismes de Placement Collectif en Valeurs Mobilières, qui permettent la gestion collective de fonds). A l'échelle internationale, les fonds de pension américains ont occupé le devant de la scène au cours de la dernière décennie, par l'importance de leurs placements financiers dans l'économie mondiale et par leur activisme en tant qu'actionnaires.

${ }^{5}$ Traduction que l'on préfère à celle de « gouvernement d'entreprise », cette dernière semblant trop restrictive, selon Roland Pérez, compte tenu de l'ampleur des dispositifs que recouvre ce nouveau principe de contrôle en termes d'institutions, de règles, de relations et de comportements (Pérez, 2003).

${ }^{6}$ Ce ratio désigne l'excèdent qui est dégagé une fois rémunérés tous les apporteurs de fonds, dettes et capitaux propres.
} 
actionnarial » suppose alors que celle-ci mette en place des politiques et des stratégies permettant de satisfaire ces contraintes de concurrence et de rentabilité du capital.

\section{Un nouveau modèle de croissance dominé par les stratégies des fusions-acquisitions, effet saillant d'une économie financiarisée}

Une internationalisation croissante des grands groupes alimente favorablement la progression de l'investissement direct à l'étranger (IDE) qui représente l'une des dimensions fortes de la globalisation et contribue à l'accroissement de la concurrence.

La tendance à l'internationalisation des entreprises de taille différente avec le développement des firmes multinationales au cours des trente dernières années ainsi que son corollaire, l'exacerbation de la concurrence, constituent une des caractéristiques de la globalisation financière. Plus précisément, à partir des années 1990 et avec la financiarisation de l'économie (détaillée plus haut) a immergé un nouveau régime de croissance adopté par la plupart des entreprises globalisées et qui répond à la logique d'un capitalisme actionnarial: il s'agit d'un nouveau modèle de croissance qui repose sur: la multiplication des fusions d'acquisition comme forme d'implantation appropriée à l'étranger, le développement d'une organisation en réseau, une orientation intensive vers le re-engineering et la sous-traitance et enfin, un recours aux restructuration des activités et aux réductions des effectifs

\section{La multiplication des fusions acquisitions}

Le premier type de stratégie qu'il semble possible de recenser concerne les procédures de fusions acquisitions des entreprises. Celles-ci consistent en une «mise en commun d'actifs ou d'intérêts économiques entre deux ou plusieurs entités » (Ceddaha, 2005, p. 7) conduisant souvent à augmenter la taille initiale des entreprises. Les opérations de fusions acquisitions ont certes été menées de façon intensive depuis le XIX ${ }^{\mathrm{e}}$ siècle. Mais de telles pratiques ont pris une dimension nouvelle depuis le début des années 1990. La diversité des secteurs d'activité touchés par les procédures de rapprochement, d'alliance, de rachat ou de cession qui ont bouleversé la structure du capital au cours de la dernière décennie permettent de mesurer l'ampleur du phénomène : l'énergie, la chimie, l'assurance, la télécommunication, la banque, la grande distribution, etc.

L'argument couramment avancé par les dirigeants pour justifier la décision de procéder à une fusion réside dans la volonté d'accéder à ce qui est appelé la «taille critique », c'est-à-dire la taille qui confère au groupe un pouvoir de marché suffisamment important pour gagner de nouvelles parts de marché et affronter la concurrence mondiale. L'autre avantage invoqué pour légitimer de telles mesures se situe dans les réductions de coûts de production offertes par les synergies résultant de ces rapprochements entre entreprises, et par les économies d'échelle que permet l'augmentation des capacités de production. Cette compression des coûts peut également s'opérer par la réduction des effectifs occupant les postes en doublon créés par la fusion entre deux entreprises exerçant la même activité (Coutinet et Sagot-Duvauroux, 2003).

Toutefois, une telle rationalité économique ne paraît pas pouvoir tout expliquer en matière de fusions acquisitions compte tenu des nombreux échecs financiers auxquels elles aboutissent parfois. Ainsi, à la logique économique rationnelle vient se mêler la rationalité politique de l'" éthos capitaliste » des actionnaires et des dirigeants, faisant de la volonté de croître et de s'étendre une raison majeure mais inavouée du choix d'entreprendre une fusion, au risque de mettre à mal l'efficacité économique - avec les bouleversements organisationnels que cela entraîne notamment -, voire même de diminuer la performance boursière (Lordon, 2002).

\section{Le développement d'une organisation en réseaux}

Dans le contexte de la configuration multinationale de la mondialisation telle que l'a définit C.-A. Michalet (2002), on voit apparaître à partir des années 1960 de grands groupes multinationaux organisés en réseaux. On parle également d'« entreprise étendue» ou d'« entreprise réticulaire » (Durand, 1999) pour désigner ce type de firme multinationale configurée selon un principe de «filialisation » ou de « satellisation » d'un certain nombre d'unités de production autour d'une maison mère. Celle-ci, habituellement appelée société holding, ou «société de participation» ou encore «société de portefeuille» (Abraham-Frois, 1996, p. 339), assure une direction commune de l'ensemble des filiales.

P. Veltz parle également d'une «PME-isation» (Veltz, 2000, p. 173) des grands groupes pour nommer ces pratiques d'externalisation et de division en unités autonomes directement orientées vers le marché, voire même pilotées par ce dernier. Cela permet à la société holding d'assigner des objectifs précis à ses entités productives et de les juger après coup. L'auteur précise qu'il existe une pluralité de formes d'entreprise réticulaire qui ne sont pas équivalentes. Il semble toutefois possible de discerner un dénominateur commun à ce qu'il appelle le «modèle cellulaire en réseau » (Veltz, 2000, p. 175). Il reposerait ainsi sur trois éléments à peu près constants : 
Tout d'abord, le fait que les unités atomisées soient dotées d'une autonomie relative à l'égard desquelles est prescrite une série d'objectifs productifs supposant un contrôle a posteriori des résultats. Ensuite, l'instauration d'une relation contractuelle entre les différentes unités productives et les entités prescriptrices et contrôleuses. Enfin, la pluralité des fonctions attribuées à ces unités, impliquant entre elles un niveau élevé de coopération.

\section{Re-engineering et sous-traitance}

Certes, la traduction stratégique de l'internationalisation des entreprises sur laquelle repose la configuration «réticulaire» des organisations productives résulterait de mesures simultanées de déconcentrations productives - c'est-à-dire d'externalisation de tout un ensemble d'activités connexes à une activité principale et un recentrage sur cette dernière - et de concentration financière, par exemple le cas avec les fusions acquisitions.

L'essor de ces concentrations financières a d'ailleurs largement bénéficié de la déréglementation et de la libéralisation des marchés financiers, ainsi que des politiques de privatisation des capitaux des entreprises. Concrètement, ces mesures se traduisent par le passage des grandes concentrations ouvrières, caractéristiques de la production de masse à de grands groupes rassemblant une kyrielle de petites unités productives représentées par les PME (Clerc, 1999). Ces externalisations peuvent notamment prendre la forme de pratiques de soustraitance.

Afin de souligner l'importance que revêt aujourd'hui une telle orientation organisationnelle typique des firmes en réseau, J.-P. Durand explique qu'auparavant, lorsque le modèle fordien prévalait, les sous-traitants n'intervenaient que sur les zones interstitielles de l'activité principale, porteuses d'une faible valeur ajoutée. Les donneurs d'ordres assumaient alors l'essentiel de la valeur ajoutée tandis qu'aujourd'hui ils « tendent à devenir seulement les concepteurs- assembleurs, qui dessinent le produit ou le service final en lui intégrant un maximum d'avancées technologiques, puis qui l'assemblent avant sa diffusion en lui donnant une marque prestigieuse porteuse d'une image valorisante » (Durand, 2004).

Les avantages que comportent les pratiques de sous-traitance du point de vue de l'entreprise globalisée sont de plusieurs ordres. Dans un contexte fortement concurrentiel, l'externalisation de la production offre tout d'abord d'importantes réductions de coûts et de délais de production. Il paraît plus intéressant de s'adresser au marché où règne une certaine concurrence plutôt qu'aux acteurs internes qui risquent de voir leur performance s'affaiblir compte tenu des marchés captifs ou monopolistiques qui sont susceptibles de s'y créer.

Le recours à la sous-traitance permet également à l'entreprise donneuse d'ordre de se dispenser de l'entretien de toute une structure de gestion de la production comprenant l'approvisionnement, le stockage, les livraisons, etc. (Durand, 2004). La réactivité et la flexibilité caractéristiques d'une relation de sous-traitance entre sous-entrepreneur et donneur d'ordre autorisent ce dernier à s'adapter rapidement à la demande et à améliorer sa compétitivité (Clerc, 1999).

Se Référant Aux Résultats D’une Enquête Consacrée Aux Chantiers De Réparation Navale, F. Ginsbourger Confirme Que L'intérêt Majeur De Ce Genre De Configuration Réside Dans Le Raccourcissement Des Délais Qui Equivaut Au Final A Des Réductions De Coûts Substantielles. L'auteur Montre Egalement Comment Les Externalisations Visent Parfois A Débarrasser Les Entreprises Des Tâches Peu Qualifiées Ou Précaires Et Cite, Pour Illustrer Cette Tendance, L'exemple De La Sous-Traitance Du Travail Dangereux Dans L'industrie Sidérurgique (Ginsbourger, 1998).

Enfin, Les Pratiques De Sous-Traitance Peuvent Egalement Revêtir Une Fonction «Politique » Consistant A «Affaiblir» Les Collectifs De Salariés Ou Bien Encore A Se Débarrasser Des Difficultés Posées Par Les Pratiques D'encadrement. Ainsi, Une Direction D'entreprise Rencontrant De Fortes Résistances Et Contestations Dans Le Management De Collectifs De Salariés De Certains De Ses Services Peut Décider De Recourir A Des Sous-Traitants. Elle Partira Du Principe Que Ces Derniers Seront Plus Faciles A « Diriger » Et A « Maîtriser » Avec La Contractualisation Qu'implique La Relation Entre Un Donneur D'ordre Et Un SousTraitant. L'entreprise Donneuse D'ordres Peut Alors Estimer Réduire Les Risques De Cette Façon En Matière De Conflits Sociaux Internes. On Souligne, A Ce Niveau, De Quelle Manière La Fonction Originelle Des Marchés Financiers Consistant A Financer Les Entreprises Est Complètement Détournée Lorsque Celles-Ci Se Décapitalisent Dans Une Perspective De Création De Valeur Financière.

\section{Les restructurations d'activité et les réductions d'effectifs}

Les restructurations d'activité et les politiques de recentrage sur les métiers de base présentent un autre type de stratégies auquel ont recours les entreprises afin de générer la «valeur actionnariale » et faire face à l'intensification de la concurrence. Celles-ci consistent à se recentrer sur les activités qui possèdent un avantage compétitif substantiel. Ainsi, les activités qui connaissent un ralentissement en raison d'une faible demande et/ou d'une concurrence accrue d'une part et d'une norme de rentabilité élevée exigée par les actionnaires 
d'autre part, seront simplement abandonnées au profit d'un recentrage sur les activités plus porteuses au regard des critères de compétitivité et de rentabilité.

Davantage encore que les fusions acquisitions, les restructurations sont à la source de nombreux licenciements, ainsi que le rappelle R. Beaujolin quand elle précise que «l'entreprise allégée réduit ses effectifs en vue de diminuer son niveau d'emploi permanent et sait se recentrer sur un noyau dur d'activités et de métiers » (Beaujolin, 1999, p. 25).

Pour qualifier les réductions d'effectifs décidées par des actionnaires peu enclins à la diversification industrielle en tant que celle-ci s'opposerait à la transparence de l'entreprise qu'implique la "gouvernance d'entreprise », ils préfèreront plutôt procéder à une diversification financière, en variant notamment la composition de leur portefeuille de participations, ce qui leur permet par la suite d'effectuer des adaptations à court terme, moins coûteuses et plus en phases avec les évolutions versatiles des marchés financiers, on parle de « licenciements boursiers » (Plihon, 2000).

\section{Conclusion}

La structure capitalistique des entreprises globalisées qui s'est progressivement transformée avec les vagues de privatisations successives depuis le milieu des années 1980 jusqu'à nos jours fut le premier indice fort qui concrétise la naissance d'un capitalisme financier. Ensuite, deux autres manifestations de l'emprise de la finance sur les organisations productives ont pu être repérées. Il s'agit d'une part des nouvelles logiques de financement auxquelles elles ont recours aujourd'hui et en particulier, le fait qu'elles utilisent davantage leurs fonds propres que l'emprunt bancaire pour se financer. D'autre part, la tendance à l'internationalisation des entreprises avec la constitution de grands groupes organisés en réseau s'est affirmée tout au long des trois dernières décennies. Cette dernière dimension a largement contribué à instituer un contexte de concurrence mondiale très élevée, entrainnant dès lors les entreprises dans une course effrénée à la compétitivité.

Compte tenu de cet environnement économique globalisé - privatisations, financement sur fonds propres et exacerbation de la concurrence internationale - les modalités de contrôle et de gestion des entreprises, ainsi que les stratégies qu'elles vont mettre en œuvre se sont, elles aussi, sensiblement transformées. Avec l'avènement du modèle d'entreprise de « shareholder » qui vient renforcer dans une large mesure le pouvoir des actionnaires, un mode inédit de contrôle des entreprises - que l'on appelle «gouvernance d'entreprise »orienté vers la maximisation de la valeur actionnariale s'instaure.

C'est dans l'objectif de satisfaire aux exigences de cette dernière que vont être mises en œuvre des stratégies telles que des fusions acquisitions, des restructurations d'activité s'accompagnant bien souvent de réductions massives d'effectifs salariés, des externalisations d'activités jugées accessoires et sous-traitées ou encore des stratégies purement financières de réduction de l'intensité capitalistique avec des pratiques de rachat d'actions.

Au final, on peut affirmer que ces stratégies qui ont pour objectif premier de réduire les coûts de production et de rendre toujours plus réactives et flexibles les unités productives ne peuvent se comprendre qu'à la lumière de l'internationalisation des entreprises - avec l'accroissement de la concurrence mondiale qui lui est constitutive - et le nouveau mode de contrôle et de direction symbolisé par la «gouvernance d'entreprise ». Et si la logique de fonctionnement optimale de l'entreprise globalisée peut être porteuse d'effets négatifs pour les salariés des entreprises et pour l'emploi, des évènements récents depuis 2000 sont révélateurs d'une crise structurelle d'un système qui amène à amplifier les inégalités et l'incertitude qui lui étaient déjà inhérentes. Cette crise ferait certes l'objet de travaux ultérieurs.

\section{Bibliographie :}

[1]. AGLIETTA Michel, La globalisation financière, L'économie mondiale, Dossiers stratégiques, CEPII, 2000, pp. $52-67$.

[2]. AGLIETTA Michel, REBERIOUX Antoine, Dérives du capitalisme financier, Paris, Albin Michel, 2004.

[3]. BATSCH Laurent, Le capitalisme financier, Paris, La Découverte, 2002, p. 121.

[4]. BAUMEL Laurent, BEAUCOURT Jean-François, DREYFUS Alain, KIERZENKOWSKI Rafal, ROUCHON Jean-Luc, « Les marchés financiers », L'économie française 2005-2006. Comptes et dossiers, Insee, 2005, pp. 198-199.

[5]. BEAUJOLIN Rachel, Les vertiges de l'emploi. L'entreprise face aux réductions d'effectifs, Paris, Grasset/Le Monde, 1999.

[6]. CABY Jérôme, HIRIGOYEN Gérard, Création de valeur et gouvernance d'entreprise, Paris, Economica, 2005.

[7]. CEDDAHA Franck, Fusions acquisitions. Evaluation, négociation, structuration, Paris, Economica, 2005.

[8]. CLERC Denis, Déchiffrer l'économie, 13e éd., Paris, Syros, 1999.

[9]. COUTINET Nathalie, SAGOT-DUVAUROUX Dominique, Economie des fusions et acquisitions, Paris, La Découverte, 2003.

[10]. COUTROT Thomas, Critique de l'organisation du travail, Paris, La Découverte, 1999.

[11]. DUMENIL Gérard, LEVY Dominique, Crise et sortie de crise. Ordres et désordres néolibéraux, Paris, PUF, 2000.

[12]. DURAND Jean-Pierre, «Chapitre I : Le nouveau modèle productif », in BOLLIER G. et DURAND C. (dir.)., La nouvelle division du travail, Paris, Editions de l'Atelier, 1999, pp. 23-42.

[13]. DURAND Jean-Pierre, La chaîne invisible. Travailler aujourd'hui : flux tendu et servitude volontaire, Paris, Seuil, 2004.

[14]. FINET Alain, «Pourquoi le gouvernement d'entreprise ? Introduction et mise en place d'un cadre théorique d'analyse », in Alain Finet (dir.), DEPRET Marc-Hubert, HAMDOUCH Abdelillah, LABIE Marc, MISSONIER-PIERA Franck, PIOT Charles (coll.), Gouvernement d'entreprise. Enjeux managériaux, comptables et financiers, Paris, De Boeck, 2005, pp. 15-38. 
[15]. GINSBOURGER Francis, La gestion contre l'entreprise. Réduire le coût du travail ou organiser sa mise en valeur, Paris, La Découverte, 1998.

[16]. INSEE, «Images économiques des entreprises et des groupes au $1^{\mathrm{er}}$ janvier 2004 », Insee Résultats, novembre 2005, $\mathrm{n}^{\circ} 23$.

[17]. LAZONICK William, O'SULLIVAN Mary, "Maximizing Shareholder Value : A New Ideology for Corporate Governance », Economy and Society, 2000, vol. 29, $\mathrm{n}^{\circ} 1$, pp. 13-35;

[18]. LORDON Frédéric, Fonds de pensions, piège à cons? Mirage de la démocratie actionnariale, Paris, Raisons d'Agir, 2000.

[19]. LORDON Frédéric, La politique du capital, Paris, Odile Jacob, 2002.

[20]. LOUE Jean-François, « Vue d'ensemble », L'économie française 2005-2006. Comptes et dossiers, Insee, 2005, pp. 7-53.

[21]. MANIERE Philippe, Marx à la corbeille. Quand les actionnaires font la révolution, Paris, Stock, 1999.

[22]. MICHALET Charles-Albert, Qu'est-ce que la mondialisation ?, Paris, La Découverte, 2002.

[23]. NIKONOFF Jacques, La comédie des fonds de pension. Une faillite intellectuelle, 2e éd., Paris, Arléa, 2000.

[24]. PEREZ Roland, La gouvernance de l'entreprise, Paris, La Découverte, 2003.

[25]. PLIHON Dominique (dir.), Rentabilité et risque dans le nouveau régime de croissance, rapport du Commissariat Général du Plan, Paris, La Documentation française, 2002.

[26]. PLIHON Dominique, Le nouveau capitalisme, Paris, La Découverte, 2004.

[27]. VELTZ Pierre, Le nouveau monde industriel, Paris, Gallimard, 2000 